\title{
Laboratory diagnosis of human infections transmitted by ticks, fleas, mites and lice in Australia
}

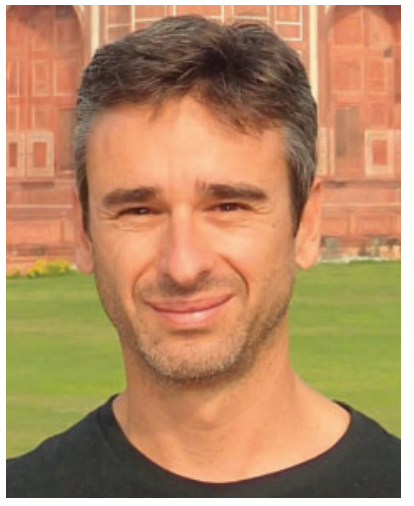

\section{John Stenos}

Australian Rickettsial Reference Laboratory, University Hospital Geelong, Vic. 3220, Australia Email: JohnS@BarwonHealth.org.au

A wide range of human pathogens (viruses, bacteria, protozoa) are transmitted by ticks, fleas, mites and lice worldwide. Some of these infections occur in Australia ${ }^{1}$, whereas others appear to be absent, although they may occur in returned travellers. The key to diagnosis is two-fold: recognition of the possibility of a vector-borne infection by the treating doctor and confirmation of the diagnosis in a diagnostic, microbiology laboratory. Laboratory diagnostic assays include culture (used rarely), nucleic acid amplification (used increasingly) and serology (used often).

In Australia the common vector-transmitted human infections (excluding those from mosquitos) are rickettsial infections, including Queensland Tick Typhus and Flinders Island Spotted Fever (from ticks), Murine Typhus and Cat Flea Typhus (from fleas) and Scrub Typhus (from mites). It is important for doctors to recognise these infections. While the patient may present with a 'viral-like illness', they actually have a bacterial infection that will respond quickly to treatment with an appropriate antibiotic, such as doxycycline ${ }^{2}$.

\section{Australian human infections transmitted by ticks, fleas, mites and lice}

\section{Viral infections}

None recognised at present but it is very likely that they exist, just not yet discovered.

\section{Bacterial infections}

(1) The main bacterial infections are rickettsial ${ }^{3}$. These consist of two genera of bacteria (Rickettsia spp and Orientia spp), which have an obligate intracellular life cycle, and many of which live

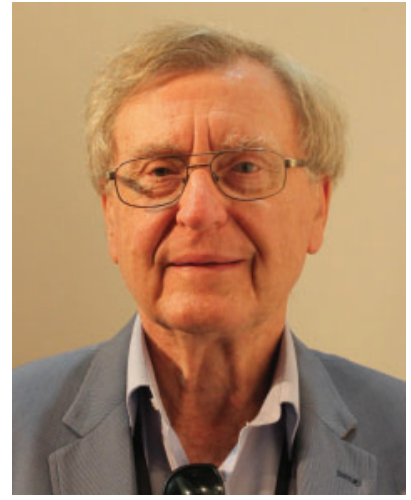

\section{Stephen R Graves}

Australian Rickettsial Reference Laboratory, University Hospital Geelong, Vic. 3220, Australia Email: Graves.rickettsia@gmail.com

in both invertebrate and vertebrate animals, moving between each with ease.

(i) Rickettsia australis is found in at least two different Australian tick species (Ixodes bolocyclus ${ }^{4}$ and I. tasmani) and various native mammals (e.g. bandicoots), causing Queensland Tick Typhus ${ }^{5,6}$ when they bite humans.

(ii) Rickettsia honei, found in the southern reptile tick Bothriocroton bydrosauri, causes Flinders Island Spotted Fever ${ }^{7-10}$. A variant of this bacterium ( $R$. honei, subspecies marmionii) found in the tick Haemaphysalis novaguinea, (and possibly others) causes Australian Spotted Fever ${ }^{11}$.

(iii) Rickettsia typhi, found in the rodent flea, causes Murine Typhus ${ }^{12-14}$, and enters humans when inoculated (by scratching the site of the flea bite) or inhaled (via dried flea faeces). The rickettsia is present in the flea faeces in very large amounts.

(iv) Rickettsia felis ${ }^{15,16}$ is found in the cat flea (which is also found on dogs). The rickettsia is present in the flea faeces.

(v) Orientia tsutsugamushi, found in Australia only in the tropical mite Leptotrombidium deliense, causes scrub typhus when the larval form of the mite (known as a 'chigger') bites humans.

(2) $Q$ Fever ${ }^{17}$, caused by the bacterium Coxiella burnetii ${ }^{18}$, can occur following the bite of an infected tick, although the most usual route of transmission is via inhalation of the dried products of parturition of an infected vertebrate animal, such as a cow, sheep or goat.

(3) Bartonella spp. comprise two human-pathogenic species, B. quintana ${ }^{19,20}$ and $B$. henselae ${ }^{21}$, arise mainly from cat bites and scratches. While it is thought that some cases may be caused by the bite of an invertebrate vector, this has not yet been confirmed in Australia.

\section{Protozoal infections}

None recognised in Australia at present although there has been an enigmatic human infection with Babesia microti ${ }^{22}$. 


\section{Human infections transmitted by ticks, fleas, mites and lice seen in overseas travellers in}

\section{Australia}

(1) African Tick Bite Fever, seen in persons returning from Africa, often having visited game parks and being bitten by an infected tick. The bacterium is Rickettsia africae ${ }^{23}$.

(2) Mediterranean Spotted Fever, seen mainly in persons returning from the Indian sub-continent. This tick-transmitted infection is caused by Rickettsia conorii ${ }^{24,25}$.

(3) Murine Typhus, caused by Rickettsia typhi, has been seen in travellers from Indonesia and elsewhere, following exposure to rodent fleas ${ }^{26}$.

(4) Scrub Typhus, caused by Orientia tsutsugamushi, is seen in travellers from various regions of Asia and Oceania ${ }^{26}$, following the bite of an infected mite and by $O$ chuto $^{27}$ in a case from Dubai. There is also evidence of scrub typhus being present in Africa and South America.

(5) Ehrlichiosis, caused by Ehrlichia chaffeensis, is seen following the bite from an infected tick in the USA ${ }^{28}$.

(6) Lyme Disease, caused by the bite of an infected tick in Europe or north America, is caused by a Spirochaete bacterium, Borrelia burgdorferi (and related species) ${ }^{29}$. This disease does not appear to be endemic in Australia and all confirmed cases in Australia to date have been in returned travellers.

Doctors in Australia should remember that malaria (a protozoal infection) and dengue (a viral infection) are more common infections in returned travellers than any of the above. They are both mosquito-transmitted infections and are still major problems.

\section{Microbiology laboratory techniques used for the diagnosis of human infections transmitted by ticks, fleas, mites and lice}

These can be divided into three groups:

(1) culture

(2) nucleic acid amplification (PCR)

(3) serology

The Australian Rickettsial Reference Laboratory (ARRL), based at University Hospital Geelong, Geelong, Victoria, uses all these modalities, depending on the actual infection being considered by the treating doctor with possible confirmation by the laboratory.

\section{(1) Culture}

Most of these specialised bacteria are obligate intracellular bacteria and must be grown in cell cultures (not agar) using cell lines that support the growth of the bacterium being sought in the patient specimen. Routine lines include mammalian (VERO, L929, DH82), amphibian (XTC2) and tick (IE6). Growth is often slow and can take many weeks, during which time the cultures need to be monitored, looking for a cytopathogenic effect (CPE) on the monolayer, or detection by nucleic amplification or antigen detection. The cell culture medium above the cell monolayer must be changed fortnightly and all work is done aseptically without antibiotics as the bacteria being sought may be susceptible to the antibiotic used. Cultures are kept for a total of six weeks. All the Rickettsia spp, Orientia spp and C . burnetii are isolated in this way ${ }^{30}$. PC-3 (BSL-3) laboratory conditions are needed to amplify these bacteria but not for the initial detection in a diagnostic modality. Culture is not useful in patient management as it is far too slow.

\section{(2) Nucleic amplification}

Real-time polymerase chain reactions (qPCR) are used routinely for detecting rickettsial ${ }^{31}$ and $C$. burnetii ${ }^{32}$ DNA in patient samples. These assays are performed on either blood in EDTA (containing the circulating leucocytes in which the bacteria are located), serum or on a biopsy of an eschar (invertebrate bite site), a rash or an operative specimen (e.g. a cardiac valve from a patient with $Q$ fever endocarditis).

Once microbial DNA has been detected, a specific microbial gene may be amplified by conventional PCR and the product sequenced to compare it with known species of microbes. 100\% homology is not always obtained as there may be polymorphisms (genetic variants) seen in some genes. Sometimes these variations are so great as to define the bacterium as a new species. Most commonly, conventional amplification cannot occur as the microbial DNA is in limited amounts and without an isolate the amplification and subsequent sequencing may be impossible to perform.

\section{(3) Serology}

This is the main diagnostic modality for this group of infections ${ }^{33}$. However interpretation is fraught with difficulties:

(i) The patient serum sample may have been taken very early in the illness before the patient's immune system has had time to produce antibodies. The serology will yield a false-negative result. A 2nd serum taken a few days/weeks later is extremely valuable diagnostically as it may now be positive for antibodies to the microbe causing the infection. This is a 'sero-conversion' and good evidence of recent infection by the microbe to which the patient has sero-converted. Alternatively, a significant rise (usually a 4-fold rise) in antibody concentration (titre) between the 1st and 2nd sera is also good evidence of recent infection.

(ii) Just because a patient has antibodies in their serum to a microbe tested for in the laboratory, it does not prove that their current illness is due to infection with that microbe. Antibodies can last for years in the patient's blood and while their presence is a marker of exposure to the microbe, it is not necessarily recent exposure. It may have been from years earlier.

(iii) A positive serology result may be a false-positive due to cross-reactivity between the patient's antibodies and a related (or even unrelated) microbial antigen being used in the laboratory assay. Using the appropriate serum screening dilution by the laboratory is crucial to prevent reporting cross-reactions as genuine positive serology results.

The ARRL uses a $1 / 128$ serum screening dilution for rickettsial serology, a 1/25 dilution for Q fever serology, 1/12 for Bartonella IgM and $1 / 64$ for Bartonella IgG and $1 / 64$ for Babesia serology screening.

These serum dilutions are based on the serology kit manufacturers' recommendations and the characteristics of the local Australian population being tested, as obtained following extensive use of 
the assays. Generally speaking, the higher the antibody titre, the more likely it is to be a genuine positive. The laboratory interpretation of the result is always important to consider.

There are many laboratory modalities for detecting antibodies (in the patient's serum) reacting with microbial antigens (in the laboratory). Enzyme immunoassay (EIA) is commonly used: complement fixation (CFT) was widely used in the past but not much now, etc. The ARRL uses mainly microimmunofluorescence (MIF) as MIF has the best sensitivity (ability to detect a genuine case) and specificity (ability to not incorrectly detect a non-case) for most of the infections transmitted by ticks, fleas, mites and lice. The laboratory should always be part of an external quality assurance program to ensure that its results are consistent with its peers. Incorrect results can lead to erroneous conclusions and inadequate management of the patient.

\section{Conflicts of interest}

The Australian Rickettsial Reference Laboratory is a human pathology (microbiology) diagnostic laboratory and Dr Stephen Graves is the Approved Pathology Provider (APP) who receives income from Medicare on a fee-for-service basis for diagnostic testing on referred patient specimens. DrJohn Stenos receives income as an employee of the laboratory.

\section{Acknowledgements}

This research did not receive any specific funding.

\section{References}

1. Graves, S.R. and Stenos, J. (2017) Tick-borne infectious diseases in Australia. Med. J. Aust. 206, 320-324. doi:10.5694/mja17.00090

2. Graves, S. (2013) Management of rickettsial diseases and Q fever.Med. Today 14 , 65-69.

3. Graves, S. and Stenos, J. (2009) Rickettsioses in Australia. Ann. N. Y. Acad. Sci. 1166, 151-155. doi:10.1111/j.1749-6632.2009.04530.x

4. Graves, S. et al. (2016) Ixodes holocyclus tick-transmitted human pathogens in north-eastern New South Wales, Australia. Trop. Med. Infect. Dis. 1, 4.

5. Wilson, P.A. et al. (2013) Queensland tick typhus: three cases with unusual clinical features. Intern. Med. J. 43, 823-825. doi:10.1111/imj.12184

6. Stewart, A. et al. (2017) Epidemiology and characteristics of Rickettsia australis (Queensland Typhus) infection in hospitalised patients in north Brisbane, Australia. Trop. Med. Infect. Dis. 2, 10.

7. Stewart, R.S. (1991) Flinders Island spotted fever: a newly recognised endemic focus of tick typhus in Bass Strait. Part 1. Clinical and epidemiological features. Med. J. Aust. 154, 94-99.

8. Stenos, J. et al. (2003) Aponomma hydrosauri, the reptile-associated tick reservoir of Rickettsia honei on Flinders Island, Australia. Am. J. Trop. Med. Hyg. 69, 314-317. doi:10.4269/ajtmh.2003.69.314

9. Unsworth, N.B. et al. (2005) Not only 'Flinders Island' spotted fever. Pathology 37, 242-245. doi:10.1080/00313020500099247

10. Raby, E. et al. (2016) New foci of Spotted Fever Group rickettsiae including Rickettsia honei in Western Australia. Trop. Med. Infect. Dis. 1, 5.

11. Unsworth, N.B. et al. (2007) Flinders Island Spotted Fever rickettsioses caused by 'marmionii' strain of Rickettsia honei, eastern Australia. Emerg. Infect. Dis. 13, 566-573. doi:10.3201/eid1304.050087

12. Graves, S.R. et al. (1992) A case of murine typhus in Queensland.Med.J. Aust. 156, 650-651.

13. Jones, S.L. et al. (2004) Murine typhus: the first reported case from Victoria. Med. J. Aust. 180, 482
14. Simon, N.G. et al. (2011) Murine typhus returns to New South Wales: a case of isolated meningoencephalitis with raised intracranial pressure. Med.J. Aust. 194, 652-654.

15. Williams, M. et al. (2011) First probable Australian cases of human infection with Rickettsia felis (cat-flea typhus). Med. J. Aust. 194, 41-43.

16. Teoh, Y.T. et al. (2017) Serological evidence of exposure to Rickettsia felis and Rickettsia typhi in Australian veterinarians. Parasit. Vectors 10, 129. doi:10.1186/ s13071-017-2075-y

17. Graves, S.R. and Islam, A. (2016) Endemic Q fever in New South Wales, Australia: a case series (2005-2013) Am. J. Trop. Med. Hyg. 95, 55-59. doi:10.4269/ajtmh. $15-0828$

18. Vincent, G. et al. (2016) Novel genotypes of Coxiella burnetii identified in isolates from Australian Q fever patients. Int. J. Med. Microbiol. 306, 463-470. doi:10.1016/j.ijmm.2016.05.014

19. Rathbone, P. et al. (1996) Bartonella (Rochalimaea) quintana causing fever and bacteraemia in an immunocompromised patient with non-Hodgkin's lymphoma. Pathology 28, 80-83. doi:10.1080/00313029600169593

20. Woolley, M.W. et al. (2007) Analysis of the first Australian strains of Bartonella quinana reveals unique genotypes. J. Clin. Microbiol. 45, 2040-2043. doi:10.1128/JCM.00175-07

21. Saisongkorh, W. et al. (2009) Emerging Bartonella in humans and animals in Asia and Australia. J. Med. Assoc. Thai. 92, 707-731.

22. Senanayake, S.N. et al. (2012) First report of human babesiosis in Australia. Med J. Aust. 196, 350-352. doi:10.5694/mja11.11378

23. Wang, J.-M. et al. (2009) Diagnosis of Queensland Tick Typhus and African Tick Bite Fever by PCR of lesion swabs. Emerg. Infect. Dis. 15, 963-965. doi:10.3201/ eid1506.080855

24. Graves, S. (2002) Imported rickettsial infections. Microbiol. Aust. 23, 33-34.

25. Punj, P. et al. (2013) A pilgrim's progress: severe Rickettsia conorii infection complicated by gangrene. Med. J. Aust. 198, 629-631. doi:10.5694/mja13.10025

26. Aung, A.K. et al. (2014) Review article: rickettsial infections in southeast Asia: implications for local populace and febrile returned travellers. Am. J. Trop. Med. Hyg. 91, 451-460. doi:10.4269/ajtmh.14-0191

27. Izzard, L. et al. (2010) Isolation of a novel Orientia species (O. chuto sp. nov.) from a patient infected in Dubai.J. Clin. Microbiol. 48, 4404-4409. doi:10.1128/ JCM.01526-10

28. Burke, A. et al. (2015) Fever and rash from Timor: where have you been and when? Med. J. Aust. 203, 338-339. doi:10.5694/mja15.00936

29. Subedi, S. et al. (2015) First report of Lyme neuroborreliosis in a returned Australian traveller. Med. J. Aust. 203, 39-41. doi:10.5694/mja14.01592

30. Vincent, G.A. et al. (2015) Isolation of Coxiella burnetii from the serum of patients with acute Q fever. J. Microbiol. Methods 119, 74-78. doi:10.1016 j.mimet.2015.10.008

31. Stenos, J. et al. (2010) Chapter 25: Rickettsia. In PCR for Clinical Microbiology (Schuller, M. ed.). Springer.

32. Stenos, J. et al. (2010) Chapter 14: Coxiella burnetii. In PCR for Clinical Microbiology (Schuller, M. ed.). Springer.

33. Graves, S. et al. (2006) Laboratory diagnosis of rickettsial infection. Aust. J. Med. Sci. 27, 39-44.

\section{Biographies}

Dr John Stenos undertook his post-doctoral studies into rickettsiae in the USA and then returned to Australia to take up appointment as the senior scientist in charge of the Australian Rickettsial Reference Laboratory, based at University Hospital Geelong, Victoria, where he has remained for the past 20 years. He is now the Research Director of the laboratory and responsible for the 'WHO Collaborating Centre for Reference and Research on Rickettsioses'.

The biography for Dr Stephen R Graves is on page 181. 